\title{
Fractal Dimension Analysis of Three-Point Bending Concrete Test Specimens
}

\author{
Jakub Sobek ${ }^{1, *}$, Petr Frantík ${ }^{1}$, Tomáš Trčka ${ }^{2}$ and David Lehký ${ }^{1}$ \\ ${ }^{1}$ Brno University of Technology, Faculty of Civil Engineering, Brno, Czech Republic \\ ${ }^{2}$ Brno University of Technology, Faculty of Electrical Engineering and Communication, \\ Brno, Czech Republic
}

\begin{abstract}
The paper deals with the analysis of the fractal dimension of fracture surfaces of concrete specimens tested in a three-point bending configuration. Fifteen representative specimens were chosen out of a bigger set for the fractal dimension analysis. Their fracture surfaces were scanned by $2 \mathrm{D}$ optical profilometry and analysed by the FracDiM software created in the Java programming language. The resulting values of fractal dimensions for specimens of three different sizes are presented.
\end{abstract}

\section{Introduction}

The aim of this paper is to analyse the geometrical properties of fracture surfaces of concrete specimens tested in a three-point bending (3PB) configuration [1]. This type of test is characterized by a higher level of constraint at the tip of a propagating crack. The presented study is a part of the research focused on the development of a complex multilevel approach to experimental-computational determination of mechanical fracture parameters of concrete, which is a typical and frequently used quasi-brittle material in civil engineering practice. A total of 42 specimens of different sizes, depths of notches and test configurations were tested in a laboratory. Half of the specimens were tested in $3 \mathrm{~PB}$ configuration with the size ratio of $1: 3$. This set consisted of specimens with three different nominal sizes and two depths of notches -or three for the smallest specimens- designated as shallow "S", middle "M" and deep "L", see Table 1. Fifteen representative specimens were chosen out of this set for fractal dimension analysis. Their fracture surfaces were scanned by $2 \mathrm{D}$ optical profilometry [2, 3, 4] and analysed by the FracDiM software [5], created in the Java ${ }^{\mathrm{TM}}$ [6] programming language. The software provides the values of fractal dimensions $D[7,8,9]$ for each scanned row, which is perpendicular to the direction of the evolving crack.

\section{Test Specimens}

A total of 15 fracture surfaces of 15 tested specimens were selected for the fractal dimension analysis. All specimens (Fig. 1) have the same breadth of $100 \mathrm{~mm}$, but they

\footnotetext{
* Corresponding author: jakub.sobek@vut.cz
} 
differ in depth and length. Nine specimens are of small size (with the depth of $100 \mathrm{~mm}$ ), three of them are of medium size $(200 \mathrm{~mm})$ and three of them are of large size $(300 \mathrm{~mm})$. The notch depths relative to the specimen depths were $0.2(\mathrm{~S}), 0.33(\mathrm{M})$ and $0.5(\mathrm{~L})$. The length of each specimen is four times its depth. Their nomenclature is listed in Table 1. The name indicates the size of the test specimen accompanied by the notch size and order number (three samples of each notch size were tested). The last letter of the name indicates which half of the fractured specimen was analysed (A or B), e.g. the specimen with nomenclature $B \_100 \_M \_2 \_B$ has a depth of $100 \mathrm{~mm}$, middle size of initial notch, it is the second specimen from the set and for fractal analysis its part B was used. All specimens were made of $\mathrm{C} 30 / 37$ concrete with maximum aggregate grain size of $16 \mathrm{~mm}$.

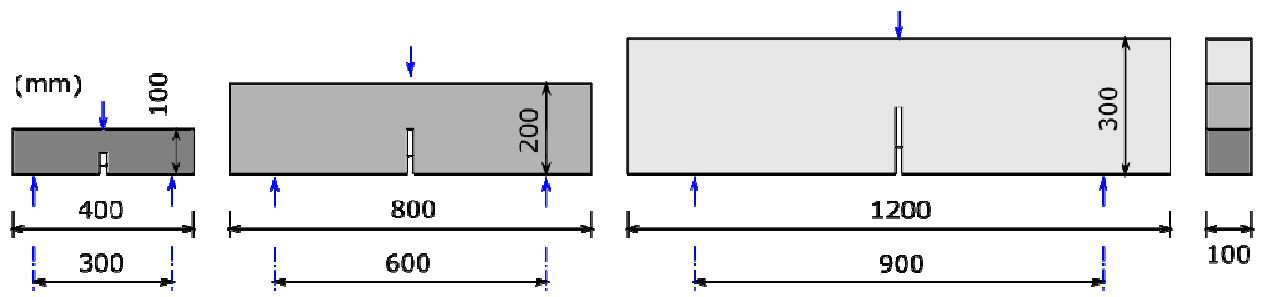

Fig. 1. Set of test specimens differing in length and depth with different initial notch sizes (notch size range is visible as white rectangle). Adopted from [10].

Table 1. Nomenclature and nominal sizes of test specimens.

\begin{tabular}{|c|c|c|}
\hline width in $\mathrm{mm}$ & notch size & nomenclature \\
\hline \multirow{9}{*}{100} & \multirow{3}{*}{ S } & B 100 S 1 A \\
\hline & & B_100_S_2_B \\
\hline & & B_100_S_3_A \\
\hline & \multirow{3}{*}{ M } & B_100_M_1_B \\
\hline & & B_100_M_2_B \\
\hline & & B_100_M_3_A \\
\hline & \multirow{3}{*}{$\mathrm{L}$} & B_100_L_1_A \\
\hline & & B_100_L_2_B \\
\hline & & B_100_L_3_A \\
\hline \multirow{3}{*}{200} & \multirow{3}{*}{$\mathrm{L}$} & B_200_L_1_A \\
\hline & & B_200_L_2_B \\
\hline & & B_200_L_3_A \\
\hline \multirow{3}{*}{300} & \multirow{3}{*}{$\mathrm{L}$} & B_300_L_1_B \\
\hline & & B_300_L_2_A \\
\hline & & B_300_L_3_B \\
\hline
\end{tabular}

\section{Optical Profilometry in 2D}

The determination of the fracture surface profiles was done by optical profilometry, which is widely used in a number of industrial applications nowadays. It is a non-destructive and non-contact method which serves for the analysis of changes in a surface plane (in a way of spatial analysis). A laser 2D profilometer generally operates with a defined wavelength semiconductor laser and uses the principle of laser triangulation for two-dimensional profile detection on different target surfaces. The diffusely reflected light from the laser line placed above the specimen is replicated on a sensitive sensor matrix by a high-quality optical system and evaluated in two dimensions. The obtained output are the values in a twodimensional coordinate system (an array which contains values of "depths" in the scanned 
plane). The distance of $50 \mu \mathrm{m}$ was selected between the scanned lines, and the spacing between adjacent points was also set to $50 \mu \mathrm{m}$ within one line. A typical illustration of the cracked ligament area during scanning is depicted in Fig. 2 where the test specimen is lying on a moving table.

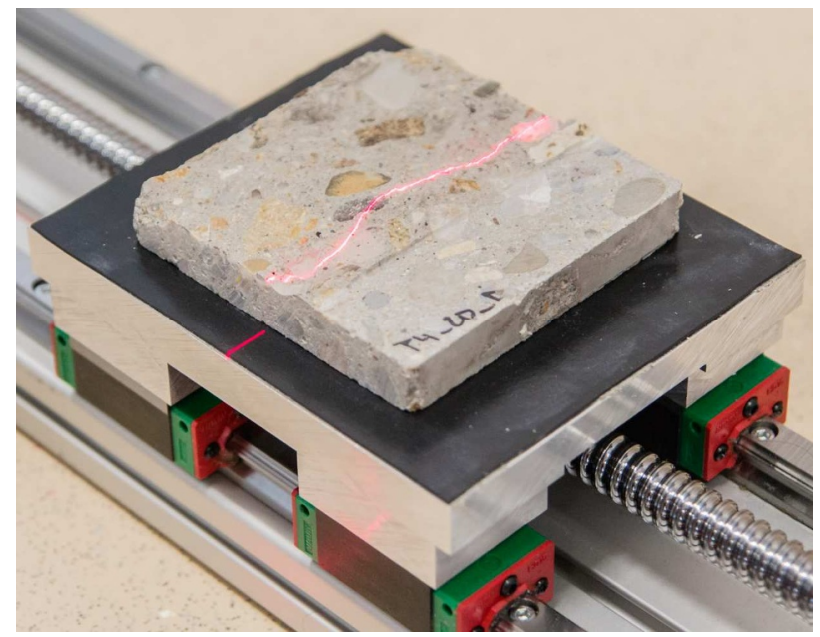

Fig. 2. Typical process of laser optical profilometry.

The image of the scanned test specimen ligament $B \_100 \_S \_3 \_A$ is shown in Fig. 3. Each row of fractal analysis of interest is parallel to $x$-axis and the rows are distanced from each other along $y$-axis by $50 \mu \mathrm{m}$.

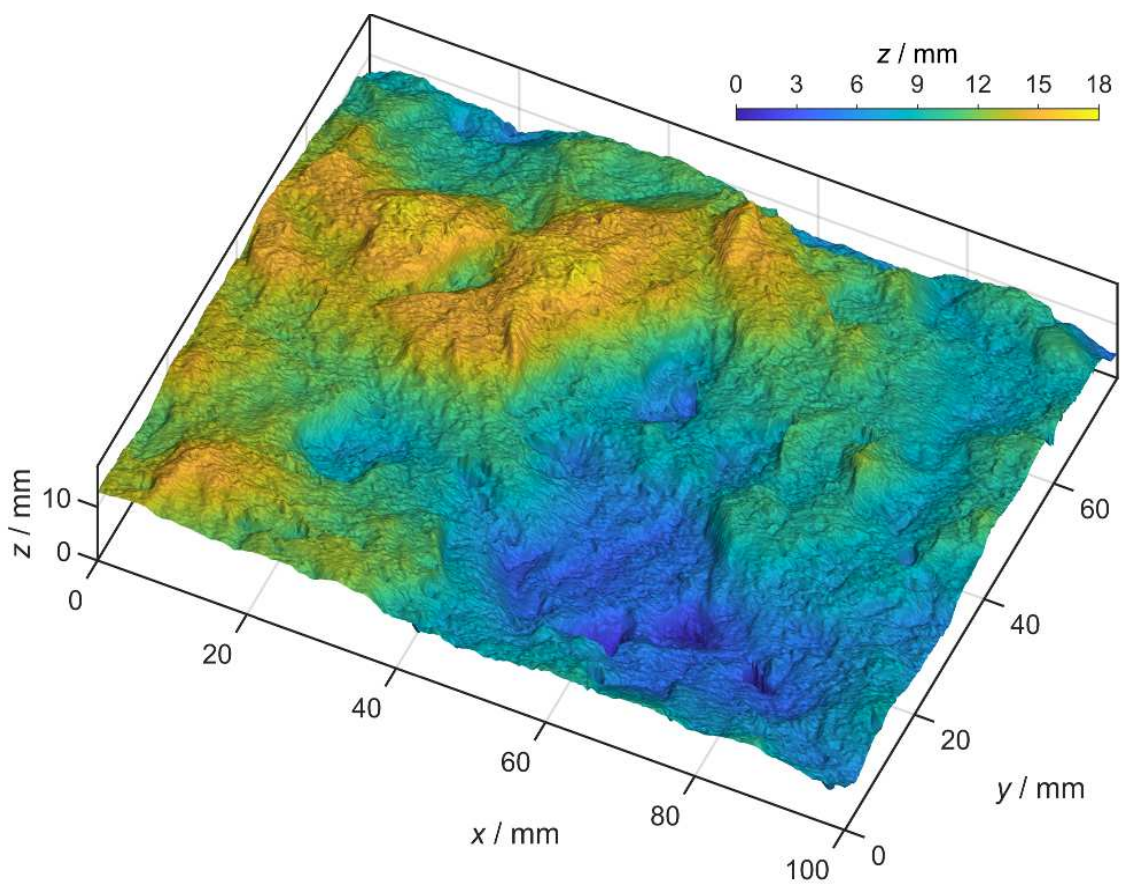

Fig. 3. Image of the scanned area of interest of ligament's profile, dimension $z$ represents depth. 


\section{Evaluation of Fractal Dimensions}

The data of all scanned lines (their depths given by $z$-axis from Fig. 3) served as inputs to the FracDiM software, developed in the Java ${ }^{\mathrm{TM}}$ programming language. Its name represents the key words Fractal, Dimension and Measurement. It serves for calculating fractal dimension values from the specimens' scanned lines. The walking divider method [11] was used together with the adjustable arc length dimension and transformation according to [7]. The software processes fractal dimensions sequentially (row by row) and is also able to provide error of its calculation. The results of the fractal analyses for all the selected specimens were compared and examined with respect to the specimen sizes and depths of notches (three different sizes). In the following section the results for specimens with the deepest notch are presented.

\section{Fractal Dimensions Discussion}

The following graphs are used (Figures 4 to 6) in order to display the differences of fractal dimensions between the individual rows of a ligament. In all three figures, the horizontal axis represents the row number. It starts at a zero value, which denotes the origin of the crack (zero value of $y$-axis in Fig. 3). The vertical axis is the value of the fractal dimension $D$ (note that the value $D=1.0$ corresponds to the fractal dimension of a line). A comparison of the results for three ligaments of the test specimens with the depth of $100 \mathrm{~mm}$ and the same notch size (the longest one) are displayed in Fig. 4. The maximum value of $D$ is 1.0615 (in the case of $B \_100 \_L \_3 \_A$, standard deviation $2 \times 10^{-4}$ ) and the minimum value is 1.0129 (in the case of $B \_100 \_L \_1 \_A$, standard deviation $1.1 \times 10^{-4}$ ). Certain sections of "peak" values for every specimen can be seen. It indicates the places where the profile of the ligament is not smooth.

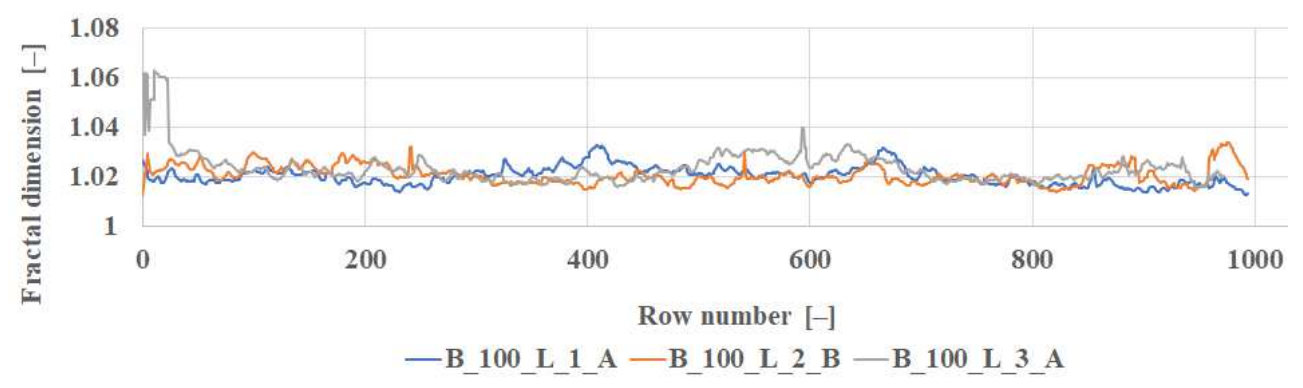

Fig. 4. Fractal dimensions of specimens with the depth of $100 \mathrm{~mm}$ and the longest notch used.

Similar trends can also be seen in Fig. 5 and Fig. 6 which depict the results for the specimens with the depths of $200 \mathrm{~mm}$ and $300 \mathrm{~mm}$ with the longest initial notch. There are a number of peaks with a higher fractal dimension. The first parts of both graphs show that the same trend and noise of $D$ values is not so high. After this region, there is a fluctuation. During three-point bending tests, stress distribution at the peak of the initial notch is completely in tension. After a crack occurs, there is a redistribution of stress and movement of the neutral axis of stress. It can change the structure of the cracked ligament. In general, although the fractal dimension values vary from row to row for different specimens, corresponding to the different shapes of their fracture process zones, the differences between specimens are relatively small. 


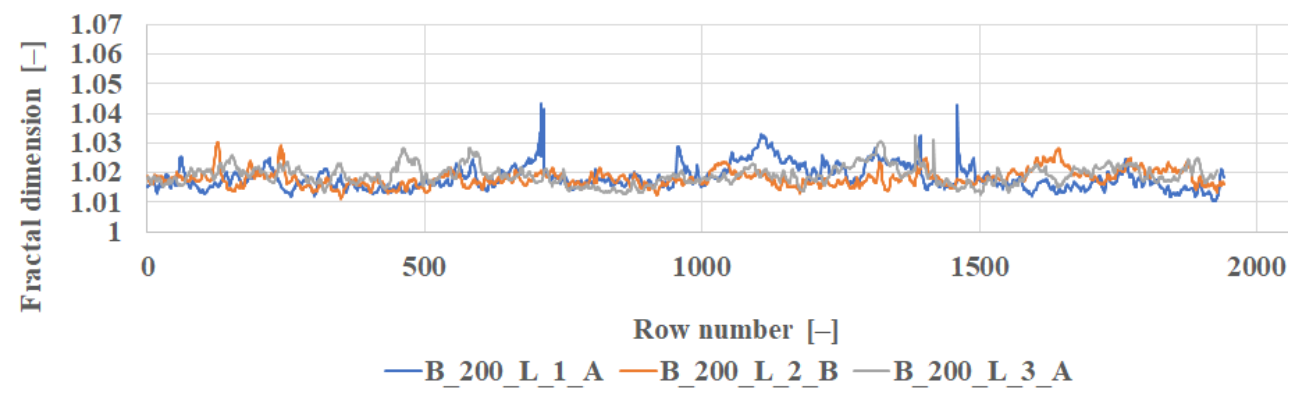

Fig. 5. Fractal dimensions of specimens with the depth of $200 \mathrm{~mm}$ and the longest notch used.

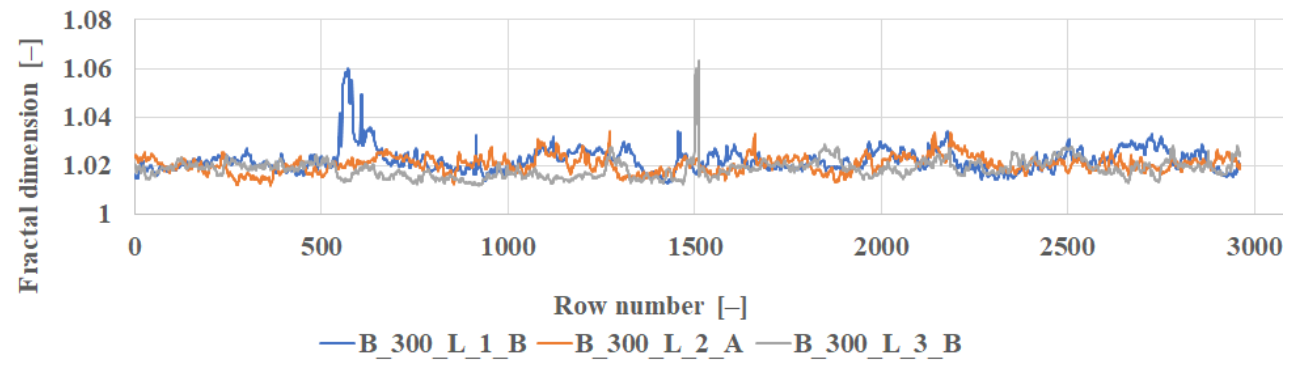

Fig. 6. Fractal dimensions of specimens with the depth of $300 \mathrm{~mm}$ and the longest notch used.

\section{Conclusions}

It was shown that it is possible to obtain the values of fractal dimensions along an evolving crack with very small standard deviations for scanned surfaces of cracked ligaments. However, no significant trend was found. Outlook of this pilot study is to add to the existing results for the rows perpendicular to the crack propagation, and also the analysis results for other directions, which may help to locate the places of interest (e.g. missing aggregate, damaged areas and degradation of material). This study opens certain venues for future research on different loading modes of test specimens with quasi-brittle matrices.

The financial support from Czech Science Foundation (GACR) project MUFRAS No. 19-09491S is gratefully acknowledged.

\section{References}

1. RILEM TC-50 FM, Determination of the fracture energy of mortar and concrete by means of three-point bend tests on notched beams, Mater. Struct. 18, 107, 287-290 (1985).

2. M. Visscher, K.G. Struik, Optical profilometry and its application to mechanically inaccessible surfaces Part I: Principles of focus error detection, Prec. Eng. 3, 16, 192198 (1994). 
3. T. Ficker, D. Martišek, Digital fracture surfaces and their roughness analysis: Applications to cement-based materials, Cem. Concr. Res. 42, 827-833 (2012).

4. P. M. D. Santos, E.N.B.S. Júlio, A state-of-the-art review on roughness quantification methods for concrete surfaces, Constr. Build. Mater. 38, 912-923 (2013).

5. P. Frantík, FracDiM software (2019), http://www.kitnarf.cz

6. Java ${ }^{\mathrm{TM}}$. Programming language (2020), http://www.java.com/en/about/

7. B. Mandelbrot, How Long Is the Coast of Britain? Statistical Self-Similarity and Fractional Dimension, Science 156, 3775, 636-638 (1967).

8. B. Mandelbrot, Les objets fractals. Forme, hasard et dimension (Flammarion, Paris, 1975).

9. A.M. Brandt, Cement-Based Composites. Materials, Mechanical Properties and Performance, Second Edition, (CRC Press, 2017).

10. D. Lehký, H. Šimonová, B. Kucharczyková, P. Daněk, Complex multilevel fracture tests of concrete: basic mechanical fracture parameters, $10^{\text {th }}$ international conference AMCM 2020, (to be published).

11. L.F. Richardson, The Problem of Contiguity: An Appendix to Statistics of Deadly Quarrels, General System Yearbook 6, 139-187 (1961). 